\title{
A preliminary analysis of the sharp-lined A3V star 95 Leo
}

\author{
Aysegul Teker ${ }^{1}$, Dursun Kocer ${ }^{1}$ and Saul J. Adelman ${ }^{2} \dagger$ \\ ${ }^{1}$ Istanbul Kultur University, Department of Mathematics and Computer Scienes, E5 Karayolu, \\ Sirinevler, 34191, Istanbul, Turkey \\ email: a.teker@iku.edu.tr, d.kocer@iku.edu.tr \\ ${ }^{2}$ Department of Physics, The Citadel, 171 Moultrie Street, Charleston, SC 29409, USA \\ email: adelmans@citadel.edu
}

\begin{abstract}
We have started an analysis of the sharp-lined superficially normal A3IVp star 95 Leo. We are using $2.4 \AA \mathrm{mm}^{-1}$ spectrograms covering $\lambda \lambda 3830-4934$ obtained with the $1.22-\mathrm{m}$ telescope of the Dominion Astrophysical Observatory using CCD detectors. Their mean signalto-noise ratios are $\geqslant 200$. The spectrograms are rectified and measured with the interactive computer graphics program REDUCE of Graham Hill and his associates. In measuring the spectrum with the VLINE routine, we use the fixed parameter feature to deconvolute metallic line blends and to measure weak lines. Effective temperature and surface gravity estimates based on spectrophotometry and $\mathrm{H} \gamma$ profile fitting were given in the literature as $8300 \mathrm{~K}$ and 3.65 , respectively.
\end{abstract}

Keywords. Line: identification, techniques: spectroscopic, stars: fundamental parameters, stars: individual: (95 Leo)

\section{Introduction}

95 Leo (HD 103578, BD+16²319, GC 16311, HR 4564, HIP 58159) is a relatively sharplined A-type spectroscopic binary star. Its spectral type is given as A3 IVp by Abt \& Morell (1995). Royer et al. (2002) note it is a SB2 binary. Adelman et al. (1980) investigated 95 Leo using UBV and ubvy photometry to obtain effective temperatures of 8650 and $8350 \mathrm{~K}$, respectively. Adelman et al. (2002) obtained $T_{\text {eff }}=8331 \mathrm{~K}, \log g=4.14$ using $u b v y \beta$ photometry and $T_{\text {eff }}=8300 \mathrm{~K}, \log g=3.65$ using spectrophotometry and $\mathrm{H} \gamma$ profile fitting.

\section{Observations and reductions}

For 95 Leo we obtained Dominion Astrophysical Observatory (DAO) $2.4 \AA \mathrm{mm}^{-1}$ SITe-2 or SITe-4 CCD spectrograms with a typical signal-to-noise ratio of 200 and a wavelength coverage of 63 or $144 \AA$, respectively. The two pixel resolution is $0.072 \AA$ which corresponds to a resolving power of 62500 . Nineteen spectrograms were obtained in the spectral range $\lambda \lambda 3830-4934$ including sections containing the $\mathrm{H} \beta$ and the $\mathrm{H} \gamma$ regions. A central stop removed light from the beam in the same manner as the secondary mirror of the telescope. We rectified the exposures with the interactive computer graphics program REDUCE (Hill \& Fisher 1986) and applied a 3.5\% correction for scattered light in the dispersion direction (Gulliver, Hill \& Adelman 1996) for many of SITe-2 spectrograms. To illustrate the program REDUCE, Figure 1 shows the normalization of the spectrogram

$\dagger$ Visiting Observer, Dominion Astrophysical Observatory, Herzberg Institute of Astrophysics, 5071 West Saanich Road, Victoria, BC, V9E 2E7, Canada 
W122_98_4020

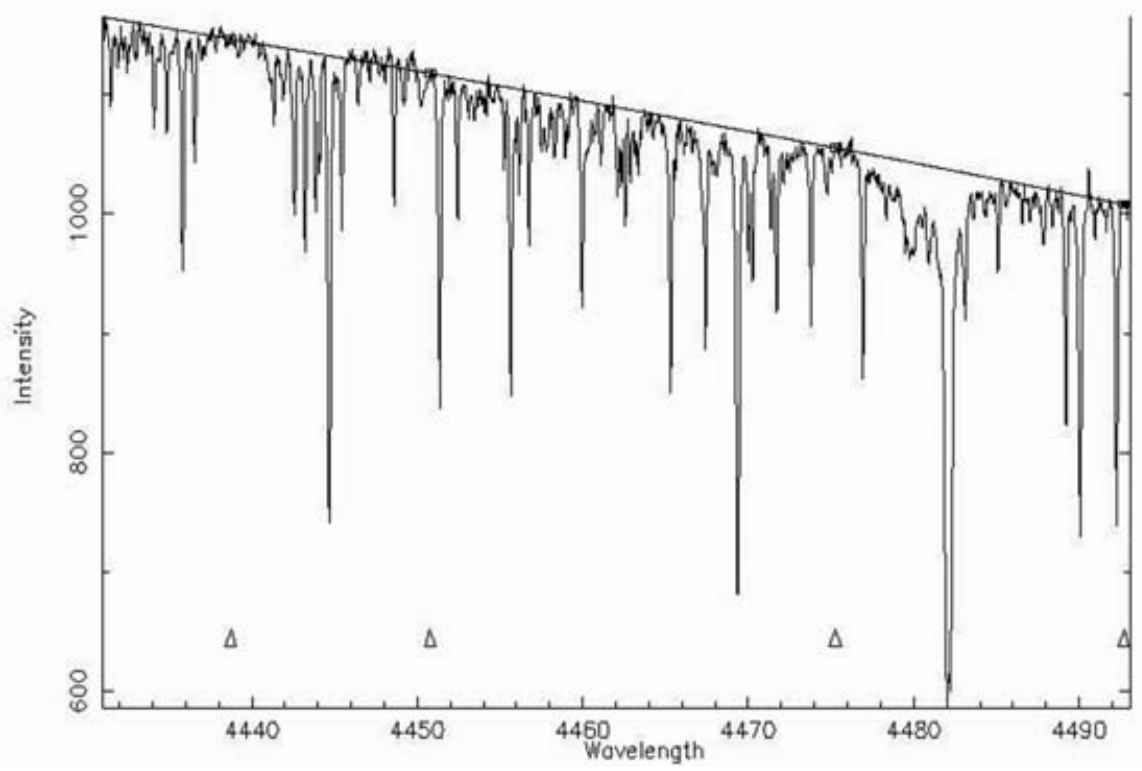

Figure 1. Normalization of the $\lambda \lambda 4430-4493$ section of the 95 Leo spectrum

covering $\lambda \lambda 4430-4493$. The radial velocities were found from the comparisons of the stellar and laboratory wavelengths after corrections were applied for the Earth's orbital velocity. The radial velocity of one spectrum was measured and we obtained a value of $-65.88 \pm 0.2 \mathrm{~km} \mathrm{~s}^{-1}$. The radial velocity of the star is given as $-21.4 \pm 0.9 \mathrm{~km} \mathrm{~s}^{-1}$ by Evans (1979).

Fix parameter mode

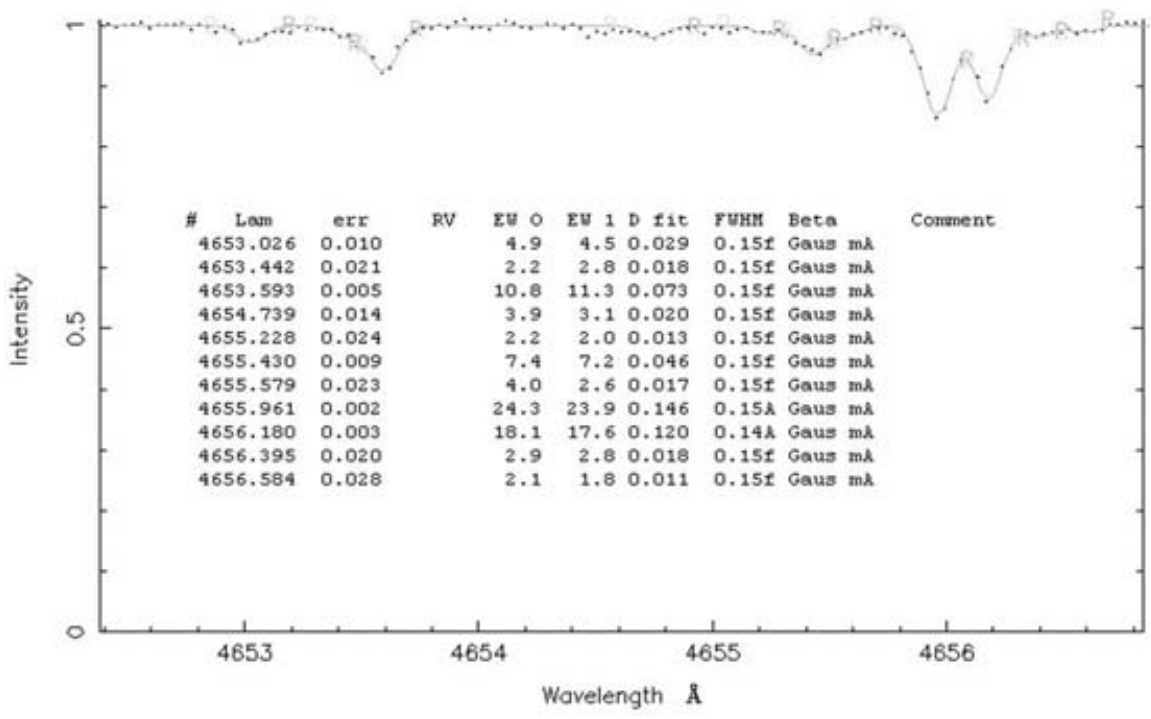

Figure 2. Measuring the spectrum of 95 Leo with VLINE 
Table 1. Line measurements of 95 Leo $(\lambda \lambda 4650-4713$ region $)$

\begin{tabular}{|c|c|c|c|c|c|}
\hline $\begin{array}{r}\text { Observed } \\
\lambda(\AA)\end{array}$ & $\begin{array}{l}\mathrm{W}_{\lambda} \\
(\mathrm{m} \AA)\end{array}$ & Depth & $\begin{array}{c}\text { Width } \\
(\mathrm{FWHM})(\AA)\end{array}$ & $\begin{array}{c}\text { Laboratory } \\
\lambda(\AA)\end{array}$ & Identification(s) \\
\hline 4653.026 & 4.5 & 0.029 & 0.146 & 4654.048 & - \\
\hline 4653.442 & 2.8 & 0.018 & 0.146 & 4654.464 & Fe I(39)4654.501(5) \\
\hline 4653.593 & 11.3 & 0.073 & 0.146 & 4654.615 & Fe I $(554,821) 4654.6050, .6286(3)$ \\
\hline 4654.739 & 3.1 & 0.020 & 0.146 & 4655.761 & Ti II(38)4655.7771(5) \\
\hline 4655.228 & 2.0 & 0.013 & 0.146 & 4656.250 & - \\
\hline 4655.430 & 7.2 & 0.046 & 0.146 & 4656.452 & Ti I(6)4656.468(25) \\
\hline 4655.579 & 2.6 & 0.017 & 0.146 & 4656.601 & - \\
\hline 4655.961 & 23.9 & 0.146 & 0.154 & 4656.983 & Fe II(43)4656.974(1) \\
\hline 4656.180 & 17.6 & 0.120 & 0.138 & 4657.203 & Ti II(59)4657.2005(33) \\
\hline 4656.395 & 2.8 & 0.018 & 0.146 & 4657.418 & Co I(156)4657.390((1)) \\
\hline 4656.584 & 1.8 & 0.011 & 0.146 & 4657.607 & $(\mathrm{Fe} \mathrm{I}(346) 4657.598((1)))$ \\
\hline 4657.828 & 5.0 & 0.032 & 0.146 & 4658.851 & - \\
\hline 4658.020 & 3.0 & 0.020 & 0.146 & 4659.043 & - \\
\hline 4660.752 & 2.6 & 0.016 & 0.146 & 4661.776 & Zr II(129)4661.78(5) \\
\hline 4662.030 & 8.0 & 0.052 & 0.146 & 4663.054 & $\mathrm{Al} \mathrm{II}(2) 4663.054(0)$ \\
\hline 4662.372 & 3.0 & 0.019 & 0.146 & 4663.396 & $\begin{array}{l}\text { Cr I(186)4663.328(40); } \\
\text { Co I(156)4663.403(12) }\end{array}$ \\
\hline 4662.681 & 14.8 & 0.095 & 0.146 & 4663.705 & $\mathrm{Fe} \mathrm{II}(44) 4663.700(0)$ \\
\hline 4662.825 & 2.7 & 0.017 & 0.146 & 4663.849 & Cr I(186)4663.832(55) \\
\hline 4663.319 & 1.8 & 0.011 & 0.146 & 4664.343 & $(\mathrm{Ni} \mathrm{I}(147) 4664.32())$ \\
\hline 4663.770 & 3.1 & 0.020 & 0.146 & 4664.794 & Cr I(186)4664.80(60) \\
\hline 4664.275 & 2.7 & 0.017 & 0.146 & 4665.299 & $($ Fe I $(1115) 4665.24())$ \\
\hline 4664.556 & 7.8 & 0.050 & 0.146 & 4665.580 & Ni II(KX)4665.548(p) \\
\hline 4664.781 & 4.2 & 0.027 & 0.146 & 4665.805 & Fe II $(26) 4665.80(p)$ \\
\hline 4664.980 & 2.3 & 0.015 & 0.146 & 4666.004 & - \\
\hline 4665.234 & 2.1 & 0.013 & 0.146 & 4666.259 & Cr I(99)4666.215(25) \\
\hline 4665.545 & 6.6 & 0.043 & 0.146 & 4666.570 & Cr I(186)4666.512(55) \\
\hline 4665.726 & 37.6 & 0.236 & 0.150 & 4666.751 & Fe II $(38) 4666.750(2)$ \\
\hline 4665.971 & 4.4 & 0.029 & 0.146 & 4666.996 & Ni I(146)4666.994(2) \\
\hline 4666.205 & 4.5 & 0.029 & 0.146 & 4667.230 & (Cr I(99)4667.181(30)) \\
\hline 4666.423 & 15.7 & 0.101 & 0.146 & 4667.448 & Fe I $(822) 4667.4531(6)$ \\
\hline 4666.653 & 4.6 & 0.030 & 0.146 & 4667.678 & $($ Ti I(6)4667.585(25)) \\
\hline 4666.797 & 4.5 & 0.029 & 0.146 & 4667.822 & Ni I(163)4667.766(3) \\
\hline 4667.105 & 12.7 & 0.082 & 0.146 & 4668.130 & Fe I $(554) 4668.1344(6)$ \\
\hline 4667.423 & 4.0 & 0.025 & 0.146 & 4668.448 & Ti I(77)4668.357(2) \\
\hline 4667.619 & 9.1 & 0.058 & 0.146 & 4668.644 & - \\
\hline 4667.890 & 4.5 & 0.029 & 0.146 & 4668.915 & $(\mathrm{La}$ II(76)4668.91(250)) \\
\hline 4668.147 & 8.9 & 0.057 & 0.146 & 4669.172 & Fe I $(821) 4669.1711((4))$ \\
\hline 4668.334 & 7.9 & 0.051 & 0.146 & 4669.359 & $\begin{array}{l}(\mathrm{Sm} \mathrm{II}(7) 4669.396(500)) ; \\
\text { Cr I(186)4669.336(50) }\end{array}$ \\
\hline
\end{tabular}

\section{Line identifications}

For each line we used the program VLINE (Hill \& Fisher 1986) to measure the equivalent width, the central wavelength, the line depth and the FWHM (full width at half maximum) of the fitted profile. The lines with equivalent widths $\geqslant 20 \mathrm{~m} \AA$ usually showed Gaussian profiles and were so fit. In measuring the spectrum with VLINE, the fixed parameter feature was applied, particulary to the line widths, as needed to better fit close blends. To illustrate the process Figure 2 shows the measurements of a spectrum section.

Our initial rotational velocity estimate based on non-blended, clearly single, mediumstrength lines with rotational profiles near $\lambda 4481$ is $4.7 \mathrm{~km} \mathrm{~s}^{-1}$. Lines with equivalent 
widths near $12 \mathrm{~m} \AA$ are sufficiantly weak to be unaffected by atomic line broadening, macroturbulance and microturbulance and are clearly on the linear part of the curve-ofgrowth. In comparison Abt \& Morrell (1995) find $v \sin i=10 \mathrm{~km} \mathrm{~s}^{-1}$ for 95 Leo using lower resolution spectra than us.

The stellar lines were identified with the general references A Multiplet Table for Astrophysical Interest (Moore 1945), and Wavelengths and Transition Probabilities for Atoms and Atomic Ions, Part I (Reader \& Corliss 1980) as well as Huldt et al. (1982) for Ti II, Iglesias \& Valesco (1964) for Mn II, Nave et al. (1994) for Fe I, and Johansson (1978) for Fe II. A sample of the line identifications is presented in Table 1 for the region centered at $4685 \AA$. Lines of Mg I, Al II, Ca I, Sc II, Ti I, Ti II, VI, Cr I, Cr II, Mn I, Mn II, Fe I, Fe II, Co I, Ni I, Ni II, Zn I, Sr II, Zr II, La II, Pr II, Nd II and Sm II were found in the one region measured so far. We anticipate being able to obtain elemental abundances for most of these species after we have found additional lines in other spectral regions.

\section{Acknowledgements}

SJA's participation was partially supported by grants from The Citadel Foundation. He thanks Dr. James E. Hesser, Director of the Dominion Astrophysical Observatory for the observing time.

\section{References}

Adelman, S. J., Pyper, D.M., \& White, R. 1980, ApJS 43, 491.

Adelman, S.J., Pintado, O.I., Nieva, F., Rayle, K.E., \& Sanders, Jr., S.E. 2002, A $8 A$ A 392, 1031. Abt, H.A., \& Morrell, N. I. 1995, ApJS 99, 135.

Evans, D.S. 1979, The Revision of The General Catalogue of Radial Velocities, IAU Symp. 30, 57.

Gulliver, A. F., Hill, G., \& Adelman, S. J. 1996, in Model Atmospheres and Spectrum Synthesis, eds. S. J. Adelman, F. Kupka, \& W. W. Weiss, ASP Conf. Ser., 108, p. 232.

Hill, G., \& Fisher, W. A. 1986, Publ. Dom. Astrophys. Obs. Victoria 16, No. 13.

Hoffleit, D. 1982, The Bright Star Catalogue, 4th revised edition, Yale University Observatory, New Haven, CT.

Huldt, S., Johansson, S., Litzen, U., \& Wyart J.-F. 1982, Phys. Scripta 25, 401.

Iglesias, L., \& Valesco, R. 1964, Publ. Inst. Opt. Madrid, No. 23.

Johansson, S. 1978, Phys. Scripta 18, 217.

Moore, C. E. 1945, A Multiplet Table of Astrophysical Interest, Princeton University Observatory.

Nave, G., Johansson, S., Learner, R.C.M., Thorne, A.P., \& Brault, J.W. 1994, ApJS 94, 221.

Reader, J., \& Corliss C. H. 1980, NSRDS-NBS 68, Part 1, US Government Printing Office, Washington, DC.

Royer, F., Grenier, S., Baylac, M.-O., Gomez, A.E., \& Zorec, J. 2002, A\&6A 393, 897. 